\title{
Prediction of Effluent Arsenic Concentration of Wastewater Treatment Plants Using Machine Learning and Kriging-Based Models
}

Mohammad Zounemat-Kermani ( $\sim$ zounemat@uk.ac.ir)

Shahid Bahonar University of Kerman

Meysam Alizamir

Islamic Azad University of Hamedan

Behrooz Keshtegar

Ton Duc Thang University

Okke Batelaan

Flinders University

\section{Reinhard Hinkelmann}

Technical University of Berlin: Technische Universitat Berlin

\section{Research Article}

Keywords: Data-mining, Hydroinformatics, Sanitary engineering, Sewage systems, WWTP

Posted Date: April 5th, 2021

DOl: https://doi.org/10.21203/rs.3.rs-353334/v1

License: (c) (i) This work is licensed under a Creative Commons Attribution 4.0 International License. Read Full License

Version of Record: A version of this preprint was published at Environmental Science and Pollution Research on November 5th, 2021. See the published version at https://doi.org/10.1007/s11356-02116916-6. 

(6)

\title{
Prediction of Effluent Arsenic Concentration of Wastewater Treatment Plants using Machine Learning and Kriging-based Models
}

\author{
Mohammad Zounemat-Kermani ${ }^{*}$, Meysam Alizamir ${ }^{2}$, Behrooz Keshtegar ${ }^{3}$, Okke Batelaan ${ }^{4}$, \\ Reinhard Hinkelmann ${ }^{5}$
}

1- Department of Water Engineering, Shahid Bahonar University of Kerman, Kerman, Iran

2- Department of Civil Engineering, Hamedan branch, Islamic Azad University, Hamedan, Iran

3- Division of Computational Mathematics and Engineering, Institute for Computational Science, Ton Duc Thang University, Ho Chi Minh City, Vietnam

4- National Centre for Groundwater Research and Training, College of Science and Engineering, Flinders University, Adelaide, Australia

5- Chair of Water Resources Management and Modeling of Hydrosystems, Technische Universität Berlin, Berlin, Germany

(*corresponding author: email: zounemat@uk.ac.ir; ORCID: 0000-0002-1421-8671)

\begin{abstract}
We evaluate the potential of kriging-based (kriging and kriging-logistic) and machine learning models (MARS, GBRT, and ANN) in predicting the effluent arsenic concentration of a wastewater treatment plant. Two distinct input combination scenarios were established, using seven quantitative and qualitative independent influent variables. In the first scenario, all of the seven independent variables were taken into account for constructing the data-driven models. For the second input scenario, we used the forward selection k-fold cross-validation method to select effective explanatory influent parameters. The results obtained from both input scenarios show that the kriging-logistic and machine learning models are effective and robust. However, using the feature selection procedure in the second scenario, made not only the architecture of the model simpler and more effective, but also enhanced the performance of the developed models. Although the standard kriging method failed to provide fair predictive results, it was revealed that the kriging-logistic method gave the best performance among the applied models.
\end{abstract}

Keywords: Data-mining; Hydroinformatics; Sanitary engineering; Sewage systems; WWTP 
$>$ Concentration of dissolved arsenic in wastewater is predicted.

$>$ Prediction efficiency of two different data-driven strategies is evaluated.

$>$ Models consist of Machine learning (MARS, GBRT, \& ANN) and kriging-based (kriging and kriging-logistic).

The kriging-logistic method gave the best performance.

40

\section{Nomenclature}

42

43 ANFIS: Adaptive Neuro-Fuzzy Inference System;

44 ANN: Artificial Neural Network;

45 As: Arsenic;

46 BF: Basis Function;

47 BOD: Biochemical Oxygen Demand;

48 COD: Chemical Oxygen Demand;

49 GBRT: Gradient Boosted Regression Trees;

50 GCV: Generalized Cross-Validation;

51 GM: Grey Model;

52 MARS: Multivariate Adaptive Regression Splines;

53 ML: Machine Learning;

54 RT: Regression Tree;

55 SS: Suspended Solids;

56 SVM: Support Vector Machine;

57 SVR: Support Vector Regression;

58 TDS: Total Dissolved Solids;

59 TSS: Total Sediment Solids;

60 WWTP: Wastewater Treatment Plant

61

62

63 


\section{Introduction}

65 Most municipal and hospital Wastewater Treatment Plants (WWTPs) have regular effluent inspections in order to meet regulation standards. However, due to high laboratory analysis costs, human mistakes, and unprecedented technical problems, etc., it is not always possible to rely on regular measurements. Under these conditions, the effluent quality cannot always be appropriately predicted using statistical and numerical methods (Pai et al., 2007; Høibye et al., 2008). In addition, the water quality of a WWTP, we focus on the arsenic (As) concentration, is sensitive to parameters such as water temperature, $\mathrm{pH}$, influent discharge, and contaminants. This is due to the fact that in WWTPs, the wastewater is biologically treated by metabolism processes resulting from the activity of microorganisms. Hence, the treatment process follows a complex and highly nonlinear trend influenced by several known and unknown independent parameters (Guo et al., 2015). In that sense, having a reliable trained mathematical system, either for data prediction in case of having missing data or checking those measured data with ambiguity in the measurement process, would be of great value.

Models based on data mining approaches, such as Machine Learning (ML) and data mining models, have already proven to be appropriate and effective tools in dealing with various environmental engineering problems (Haghiabi et al., 2018; Zounemat-Kermani et al., 2020). Given their architecture, ML models are capable of predicting a nonlinear systems' output value(s) just relying on the input parameters after the ML models have been trained and evaluated.

Due to the complication of the wastewater treatment processes, several researchers have already attempted to evaluate the effectiveness of machine learning models in modeling and predicting water quality in WWTPs effluent (Comas et al., 2001; Qin et al., 2012; Zounemat-Kermani et al., 2019; Bernardelli et al., 2020; Icke et al., 2020). Pai et al. (2007) used Grey Model (GM) and Artificial Neural Network (ANN) to predict Chemical Oxygen Demand (COD) and Suspended Solids (SS) in a hospital effluent from a wastewater treatment plant. They confirmed the suitability of ANN application in the prediction process. In another similar study, Pai et al. (2009) developed machine learning methods including Adaptive Neuro-Fuzzy Inference System (ANFIS) and ANN for the effluent prediction of SS and COD of a hospital wastewater treatment plant. It was found that the employed ANFIS model acted better in the prediction of the effluent parameters. Guo et al. (2015) employed ANNs and Support Vector Machines (SVMs) to predict the effluent concentration in a WWTP in Korea. Both models acted acceptably in the prediction procedure; however, it was found that the SVM model surpassed the ANN in terms of models' accuracy. Sharafati et al. (2020) applied ensemble ML models, including the AdaBoost regression, gradient boost regression, and random forest regression, in predicting the WWTP effluent water quality parameters, such as Total Dissolved Solids (TDS), COD, and Biochemical performance in the prediction process of TDS and COD, respectively. Granata et al. (2017) 
attempted to model and estimate the wastewater quality such as BOD, COD, TSS, and TDS indicators using support vector regression (SVR) and Regression Tree (RT) as black-box indirect modeling. They reported successful application of both machine learning models signifying that the SVR model acted better than the RT model. Ansari et al. (2018) modeled and predicted the influent flow rate of a sewage treatment plant using stochastic, nonlinear autoregressive network and SVM model. The results of the study demonstrated that the SVM model was successful in modeling peak inflow. In a study done by Fard et al. (2020), multilayer perceptron ANNs trained by the Levenberg-Marquardt algorithm and genetic algorithm was employed to predict the total COD, ammonia, phosphate and turbidity of the effluent of slaughterhouse WWTP. The finding of the study indicated that ANN trained by the genetic algorithm performed better than the standard ANN.

Arsenic in water resources is highly toxic, and is a global occurring problem. Thus, different strategies have been employed to remove arsenic or diminish concentration levels in drinking water, industrial and wastewater effluent (Mohan and Pittman, 2007; Sheikhi et al., 2021). Arsenic removal technologies in WWTPs and other facilities cover a wide range of methods, such as oxidation (Zhang et al., 2018), coagulation and softening (Ge et al., 2020), sorption and ion-exchange techniques (Zhou et al., 2017), synergistic reduction (Wang et al., 2020), membrane technologies (Hubadillah et al., 2019), and bioremediation (Plewniak et al., 2018). Despite that fact that there are not any related works in using ML models to model/predict effluent arsenic in WWTPs, several researchers developed ML approaches to model the removal process of arsenic in fields of chemical and environmental engineering. Instances include using ANNs in the prediction of the arsenic removal from aqueous solution by living cells of Bacillus cereus biomass (Giri et al., 2011), using an ANFIS model to simulate the removal of arsenic and chromium from water (Mandal et al., 2015), using ANNs to model the removal of aquatic arsenic species by indigenous microbes (Altowayti et al., 2020).

This study aims to predict the effluent arsenic concentration in WWTPs based on several influent water quality parameters, such as influent arsenic values, flow, BOD, TSS, pH, temperature, and turbidity, using data-driven techniques, including kriging, logistic-kriging, multi-layer perceptron ANN, Gradient Boosted Regression Trees (GBRT), and Multivariate Adaptive Regression Splines (MARS). To the best of the authors' knowledge, previous studies have not implemented statistical and ML methods to model effluent arsenic concentration in WWTPs. Moreover, pertinent studies in the field of arsenic removal modeling using ML methods are confined to conventional and simple soft computing methods such as ANNs and ANFIS. Accordingly, the present study's contribution not only comprises the specific case study problem but also challenges the capability of five different data mining statistical (kriging and kriging-logistic) and ML (ANN, GBRT, and MARS) methods in the prediction procedure. 
156

\section{Case Study and Dataset Description}

In this study, 10 years of monthly quantity and quality influent (input parameters) and effluent (target parameter) have been used for the development of the models. The input vector consists of influent variables, including As concentration, flow, BOD, Total Sediment Solids (TSS), pH, water temperature, and turbidity. On the other hand, the output (target) parameter is the effluent arsenic concentration. All datasets were extracted from the annual pretreatment reports for the Point Loma wastewater treatment plant, San Diego City, for 2009 to 2109. The Point Loma WWTP opened in 1963 and treats almost 660,000 cubic meters of wastewater per day. The reports include a summary of pretreatment program activities of the metropolitan sewerage system, including the Point Loma WWTP, the South Bay Water Reclamation Plant, and the North City Water Reclamation Plant (Pretreatment Annual Report for the Point Loma POTW, 2009-2019). Figure 1 illustrates the location of the Point Loma WWTP. Table 1 summarizes the statistical characteristics of the data used in this study. One interesting fact about the mean values presented in Table 1 is that, in general, the WWTP has mitigated the effluent arsenic by $30 \%$ (from $1.42 \mathrm{ug} / \mathrm{L}$ to $0.99 \mathrm{ug} / \mathrm{L}$ ).

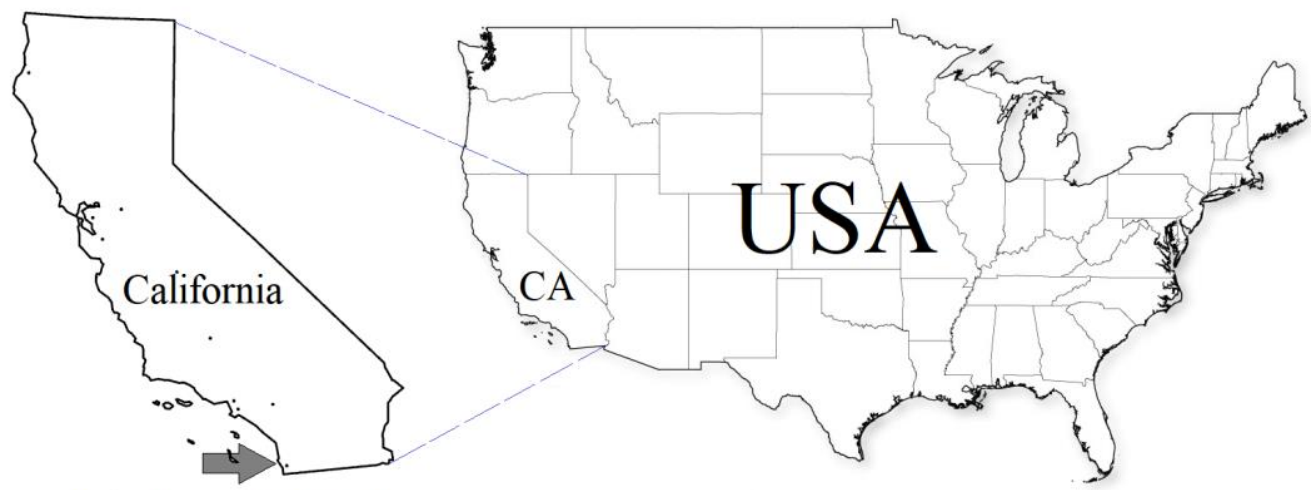

Point Loma WWTP

Figure 1. Location of the Point Loma WWTP

\begin{tabular}{lllllllll}
\hline Type & Variable & Mean & St.Dev & $\mathrm{CV}^{*}$ & Minimum & Maximum & Kurtosis & Correlation** \\
\hline \multirow{6}{*}{ Input } & Flow rate $\left(\mathrm{m}^{3} / \mathrm{s}\right)$ & 6.27 & 0.50 & 8.01 & 5.55 & 7.96 & 1.14 & -0.20 \\
& BOD-Inf $(\mathrm{mg} / \mathrm{L})$ & 313.94 & 22.58 & 7.19 & 241.00 & 362.00 & 0.32 & 0.22 \\
& TSS $(\mathrm{mg} / \mathrm{L})$ & 347.63 & 25.19 & 7.24 & 263.00 & 406.00 & 0.64 & 0.30 \\
& $\mathrm{pH}$ & 7.36 & 0.07 & 0.93 & 7.20 & 7.50 & -0.74 & -0.32 \\
& Temperature $\left({ }^{\circ} \mathrm{C}\right)$ & 25.58 & 1.85 & 7.22 & 22.20 & 28.70 & -1.24 & 0.31 \\
& Turbidity $(\mathrm{NTU})$ & 133.30 & 15.63 & 11.73 & 104.00 & 182.00 & 1.02 & -0.21 \\
& Arsenic-Inf. (ug/L) & 1.42 & 0.45 & 31.95 & 0.50 & 3.55 & 3.47 & 0.89 \\
\hline Output & Arsenic-eff. (ug/L) & 0.99 & 0.35 & 35.65 & 0.35 & 2.69 & 3.92 & 1.00 \\
\hline
\end{tabular}


Note: * Coefficient of variation, ** Pearson's correlation coefficient between the input parameter and effluent arsenic

Besides, based on the highest Pearson's correlation coefficient provided in Table 1, Figure 2 depicts the annual variations of the influent and effluent arsenic concentration using boxplots. As shown in Figure 2, the effluent arsenic follows the general variational trend of the influent arsenic values. Moreover, it can also be realized that both influent and effluent arsenic concentrations have recorded new maximum values in 2018 and 2019.

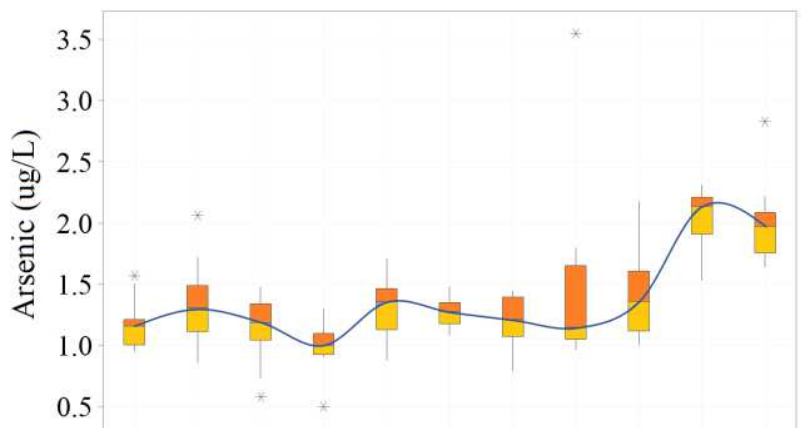

(a)

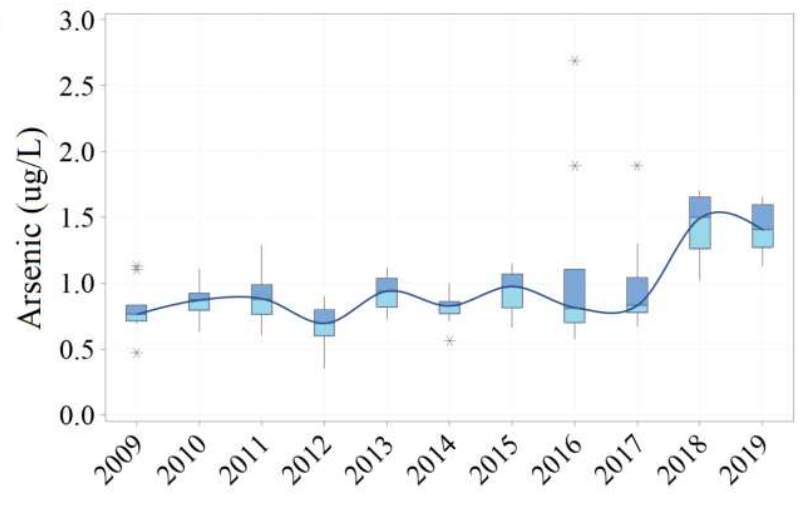

Figure 2. Annual variations of the arsenic concentrations using boxplots based on the monthly datasets; (a) influent and (b) effluent; (asterisks represent the outlier values)

\section{Methods}

In this section, the methodology and philosophy behind developing the GBRT, MARS, and kriging-based models are described. The results of these models are later compared with the conventional and well-known perceptron ANN, as a benchmark model, for evaluating the capability of the applied ML and kriging-based models. It should be noted that for details on the perceptron ANN method we refer to numerous available papers and reports explaining the perceptron ANN, such as Gardner and Dorling (1998) and Zounemat-Kermani et al. (2009).

\section{Gradient Boosted Regression Trees (GBRT)}

GBRT is one of the most widely applied techniques for regression-based problems (Friedman, 2001). The core concept of the GBRT is based on improving the Regression Tree (RT) performance by employing the most explanatory input variables to reach the most accurate corresponding target. Generally, the RTs generate several leaf nodes, by mapping the predictor features to target values, that make several advantages like easy to find the interaction between input and response parameters, comprehensive structure to analyze the obtained results, and finally, computationally efficient (Song et al., 2019; Samadi et al., 2021). In the GBRT approach, generating initial RTs is carried out as the first step. Then, the RTs are trained through multiple 
187 iterations to minimize residual values. The forward stage format of the GBRT can be described 188 as follows:

$$
\mathrm{F}_{\mathrm{m}}(\mathrm{x})=\mathrm{F}_{\mathrm{m}-1}(\mathrm{x})+\mathrm{h}_{\mathrm{m}}(\mathrm{x})
$$

where $\mathrm{F}(\mathrm{x})$ and $\mathrm{h}(\mathrm{x})$ denotes the GBRT model and basis function, respectively. Also, $\mathrm{m}$ and $\mathrm{x}$ represent boosting iteration and input parameters. By finding the optimum value for $h(x)$, response values can be obtained using the following equation:

$\mathrm{F}_{\mathrm{m}}\left(\mathrm{x}_{\mathrm{i}, \mathrm{t}}\right)=\mathrm{F}_{\mathrm{m}-1}\left(\mathrm{x}_{\mathrm{i}, \mathrm{t}}\right)+\mathrm{h}_{\mathrm{m}}\left(\mathrm{x}_{\mathrm{i}, \mathrm{t}}\right)=\mathrm{Y}_{\mathrm{i}, \mathrm{t}+\mathrm{k}}$

where $\mathrm{Y}_{\mathrm{i}, \mathrm{t}+\mathrm{k}}$ indicates the target value.

After each iteration, a new RT is fitted to reach a minimum error for the training phase. To improve the generalization performance by avoiding the over-fitting problem, the GBRT model applies the learning rate factor $(\eta)$ to set the contribution of each RT in the final model by considering the number of iterations (Hastie et al., 2009; Song et al., 2019; Alizamir et al., 2020a). After employing this factor, the main equation can be presented as follows (Kim et al., 2020):

$\mathrm{F}_{\mathrm{m}}(\mathrm{x})=\mathrm{F}_{\mathrm{m}-1}(\mathrm{x})+\eta \mathrm{h}_{\mathrm{m}}(\mathrm{x}) \quad \eta \in[0 ; 1]$

Smaller values of $\eta$ helps the model to perform more accurate during the testing phase; however, in this case, the model needs more iterations as well. In this study, parameters of the GBRT model were tuned based on trial-and-error process to minimize difference between the target and actual values. In addition, the shrinkage factor, subsampling factor, and maximum tree depth were set to $0.015,0.25$, and 5 , respectively. Also, 150 number of iterations and the squared loss function were applied for the training process. More detail on the structure and the training procedure of the GBRT can be found in Friedman (2001).

\section{Multivariate Adaptive Regression Splines (MARS)}

MARS was proposed by Friedman (1991) as a robust nonparametric statistical tool to extract the relationship between the response and input parameters. The MARS model, by dividing the training dataset into splines and slope values, constructs a linear basis function. After fitting a regression equation to the training dataset, Basis Functions (BFs) and knots can be obtained (Heddam and Kisi, 2018). The MARS algorithm achieves more error reduction by employing a forward phase sets location of each knot and its corresponding pair of the BFs. Also, the backward phase improves the performance of the MARS models by deleting the redundant BFs that have negligible effects on the model using the Generalized Cross-Validation (GCV). The general expression of a MARS model can be presented as follows (Zhang and Goh, 2016; Alizamir et al., 2020b): 
$221 \quad \mathrm{f}(\mathrm{x})=\beta_{0}+\sum_{\mathrm{m}=1}^{\mathrm{M}} \beta_{\mathrm{m}} \lambda_{\mathrm{m}}(\mathrm{X})$

222

$\operatorname{GCV}(M)=\frac{\frac{1}{n} \sum_{i=1}^{N}\left(y_{i}-f\left(x_{i}\right)\right)^{2}}{\left(1-\frac{c(M)}{n}\right)^{2}}$

223 where $\mathrm{f}(\mathrm{x})$ stands for the dependent variable. $\lambda_{\mathrm{m}}$ and $\beta$ are respectively a spline function and a

224 constant coefficient calculated by using the least square methodology. Also, a linear function in

225 the MARS model can be defined at value $t$ as follows:

226

$\max (0, x-t)=\left\{\begin{array}{cc}x-t, & \text { if } \quad x \geq t \\ 0, & \text { otherwise }\end{array}\right.$

227 Finally, the GCV value indicated the best-fitted model. For this study, the GCV value and 228 highest degree of interactions in the final model were equal to 0.0128 and 1, respectively. More 229 details regarding MARS are available in Friedman (1991) and Friedman and Roosen (1995).

230

231

232

233

234

235

236

237

238

239

240

241

242

243

244

245

246

247 and

248

\section{Kriging-based Methods}

The Kriging-based surrogate model was introduced by Krige (1952), while Matheron (1963) mathematically formulated the Kriging model. Commonly, the Kriging models can be applied for the prediction of complex problems such as hydrological fields (Heddam et al., 2019), structural design optimization (Sakata et al., 2003, Huang et al., 2006), energy (Keshtegar et al., 2018), and structural reliability analysis (Yun et al., 2018; Jiang et al., 2020) due to its ability in handling the high-nonlinear relations.

In the standard and conventional kriging model, two terms are used for the interpolation of a mathematical relation as follows:

$P(X)=G(X)^{T} \beta+r(X)^{T} \gamma$

In the first term of Equation $7, G(X)$ and $\beta$ respectively denote the Basis Function (BF) and unknown coefficient vectors. The nonlinearity of the predicted model is controlled by the nonlinear form of function $G(X)$. When $G(X)^{T} \beta$ has the accurate tendency for training phase, the accuracy of the model is increased in the testing phase. For accurate predictions with highest tendency and agreement, the BF mathematical form should be selected on the tended line of the prediction model. The second term represents the stochastic term, where

$\gamma=R^{-1}\left(O-G^{T} \beta\right)$

$r(X)=\left[R\left(\theta, X_{1}, X\right), R\left(\theta, X_{2}, X\right), \ldots, R\left(\theta, X_{n}, X\right)\right]^{T}$ 
In Equations (8) and (9), $\mathrm{O}$ represents the observed data in the training phase. $R$ denotes the correlation function, which is given a stationary Gaussian function with the input of $X_{p}, X_{q}$ (p, q $=1,2, \ldots, m$ ) (where $\mathrm{p}$ - $t h$ and $\mathrm{q}$-th input samples for $\mathrm{m}$ data points) and correlation parameter $\theta$. The parameter $\theta$ is determined by using the maximum likelihood estimator as follows (Lu et al., 2018):

$$
L(\theta)=-m\left(\operatorname{Ln}\left(\sigma^{2}\right)+\operatorname{Ln}(|R|)\right.
$$

In Equation (10), $L n$ represents the logarithmic operator. The correlation matrix, $R$, is determined as below:

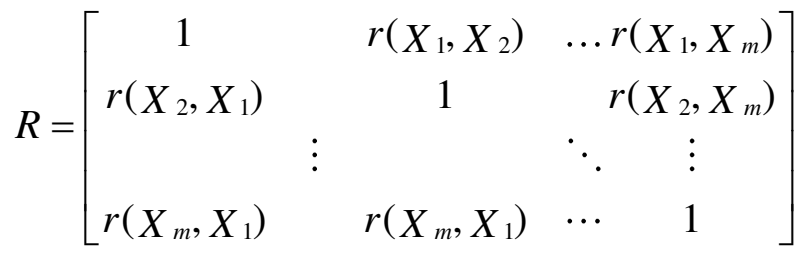

In which $\mathrm{r}\left(X_{p}, X_{q}\right)$ is the covariance basis function. Commonly, the Gaussian function is utilized to define the kernel function in the modeling process (Sun et al., 2017). By applying the Gaussian function for the correlation function, $r\left(X_{p}, X_{q}\right)$ is given as follows:

$$
r\left(X_{p}, X_{q}\right)=\exp \left[-\sum_{i=1}^{k} \theta^{i}\left(X_{p}^{i}-X_{q}^{i}\right)^{2}\right]
$$

where $\theta^{i}, i=1,2, \ldots, k$ (where $\mathrm{i}$-th correlation parameter for $k$ input variables). The parameters of Equation (7) using the optimum condition of Equation (10) are given as follow: $\beta=\left(G^{T} R^{-1} G\right)^{-1} G^{T} R^{-1} O$

By comparing the Kriging model, the BF is affected by the parameters of the Kernel function in the stochastic term. Therefore, in the regression analysis of problems with limited database, the BFs are effective tools to provide accurately predicted results. Commonly, the second-order polynomial function is used for the Kriging model by the following relation:

$$
G(X)^{T} \beta=\beta_{0}+\beta_{1} x+\beta_{2} x^{2}
$$

The two-terms in the Kriging interpolation provide the perfect prediction in the training phase for the studied data set. Nonetheless, the abilities of the predicted result for the testing (validation) dataset for both accuracy and tendency are dependent on the BFs. In nonlinear prediction, the accuracy of the problem can be improved based on the flexible function, which can be changed from linear to nonlinear forms. Thus, the logistic function with sigmoid form can predict a nonlinear function with high-flexibility in the test phase by the following relation:

$$
G(X)^{T} \beta=\beta_{0}+\beta_{1} x+\frac{\beta_{2}}{1+\exp (-x)}
$$

The logistic function presented in Eq. (15) with sigmoid form can predict a nonlinear function using factor $\kappa$ which is determined by trial and error in the current work. The presented function in Equations (14) and (15) are used in the Kriging model and the Kriging-based Logistic models, which are proposed in this study. As can be realized, the presented logistic model is as simple as 
281 the standard Kriging model, and it has all the abilities of the standard Kriging models in the 282 training phase in achieving perfect simulations.

283 The models presented in Eqs. (14) and (15) can be used for predicting the data in the testing 284 (validation) phase under the input database as below:

285 Step 1: Determining $r$ using Eq. (9) for point $\boldsymbol{X}$;

286 Step2: Computing stochastic term $r(X)^{T} \gamma$ using the attained results from Step 1;

287 Step 3: Computing $G(X)^{T} \beta$ for the point $\boldsymbol{X}$ using Eqs. (13) and (14);

288 Step 4: predicting target data using Eq. (7)

289 As can be seen, the unknown coefficients and correlation parameters are computed in the 290 regression process using the Eqs. (10) and (13) for the training data points. The correlation 291 matrix, $R$, applied in Eqs. (8) and (13) is computed for the training data while the $r(X)$ is 292 determined as presented in step 1 for the testing data points.

293 The prediction in the testing dataset of these models should be evaluated based on several 294 statistical models for accuracy, tendency, and agreement. We believe that this model could 295 provide the most accurate results for problems with limited data points in the training phase 296 subject to the number of training data being more than $2 k+1$.

\section{Application of Models}

298 Feature selection process

299 Besides the importance of methods used for achieving proper prediction/simulation results in 300 data mining problems, feature selection - which is the process of choosing the best and most relevant input parameters - plays an essential role in constructing the optimum data mining model. In other words, in the feature selection process, one confines the original input vector to an optimal one by eliminating some irrelevant or non-effective input parameters, which leads to a faster training procedure and more accurate results. There are a variety of different approaches for applying the feature selection process, including the wrapper methods (e.g., forward feature selection) (Meyer et al., 2018), Filter methods (e.g., the mutual information approach) (Cameron et al., 2019), and embedded methods (Rodriguez-Galiano, 2018).

In this study, based on the wrapper methodology, we used the forward selection combined with the k-fold cross-validation method, where ten folds were randomly assigned for applying the technique. The result of the feature selection process implies that just three input parameters, i.e., influent flow, influent temperature, and influent arsenic values, have an effective influence on

312 the target value (effluent arsenic). In Figure 3, the final results of the feature selection process, 313 based on the calculated R-squared (\%) for all of the input parameters and the selected 314 parameters, is illustrated. 


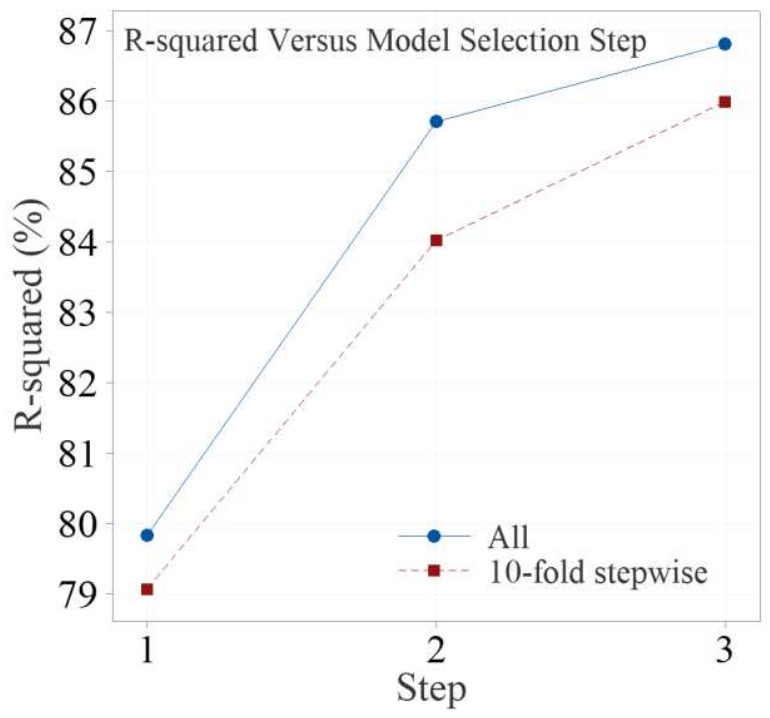

Figure 3. The result of forward feature selection analysis with 10 -fold cross-validation leading to select 3 effective input parameters (flow rate, influent arsenic, and temperature) out of the 7 available variables (see Table 1 )

\section{Modeling scenarios}

This study deals with two different types of input combinations for constructing the data mining models; in the first scenario, all of the seven available independent variables are considered as the input vector (see Table 1). On the other hand, the second scenario is established based upon the effective independent variables elicited from the feature selection process; flow rate, influent arsenic, and temperature. Differentiating between these two input scenarios would help to evaluate the effectiveness and significance of the feature selection process on the final performance of the applied models in predicting effluent arsenic.

\section{Evaluation of the models' performance}

To evaluate the performance of the models applied, several statistical measures are used, including the Root Mean Square Error (RMSE) and Mean Absolute Error (MAE), as absolute scale deviance measures, the Mean Absolute Geometric Error (MAGE) and Geometric Reliability Index (GRI), as relative scale deviance measures, the Pearson's Correlation Coefficient (PCC) and Nash-Sutcliffe Efficiency (NSE) coefficient, as similarity measures (Jachner et al., 2007). The formula for each of the above-mentioned measures, as well as the possible ranges and ideal values, are presented in Table 2.

Table 2. Statistical measures used in this study for the evaluation of models applied

\begin{tabular}{lll|l}
\hline $\begin{array}{l}\text { Classification } \\
\text { of measures }\end{array}$ & $\begin{array}{l}\text { Statistical } \\
\text { measure }\end{array}$ & Formula & Range \& Ideal value(s) \\
\hline
\end{tabular}




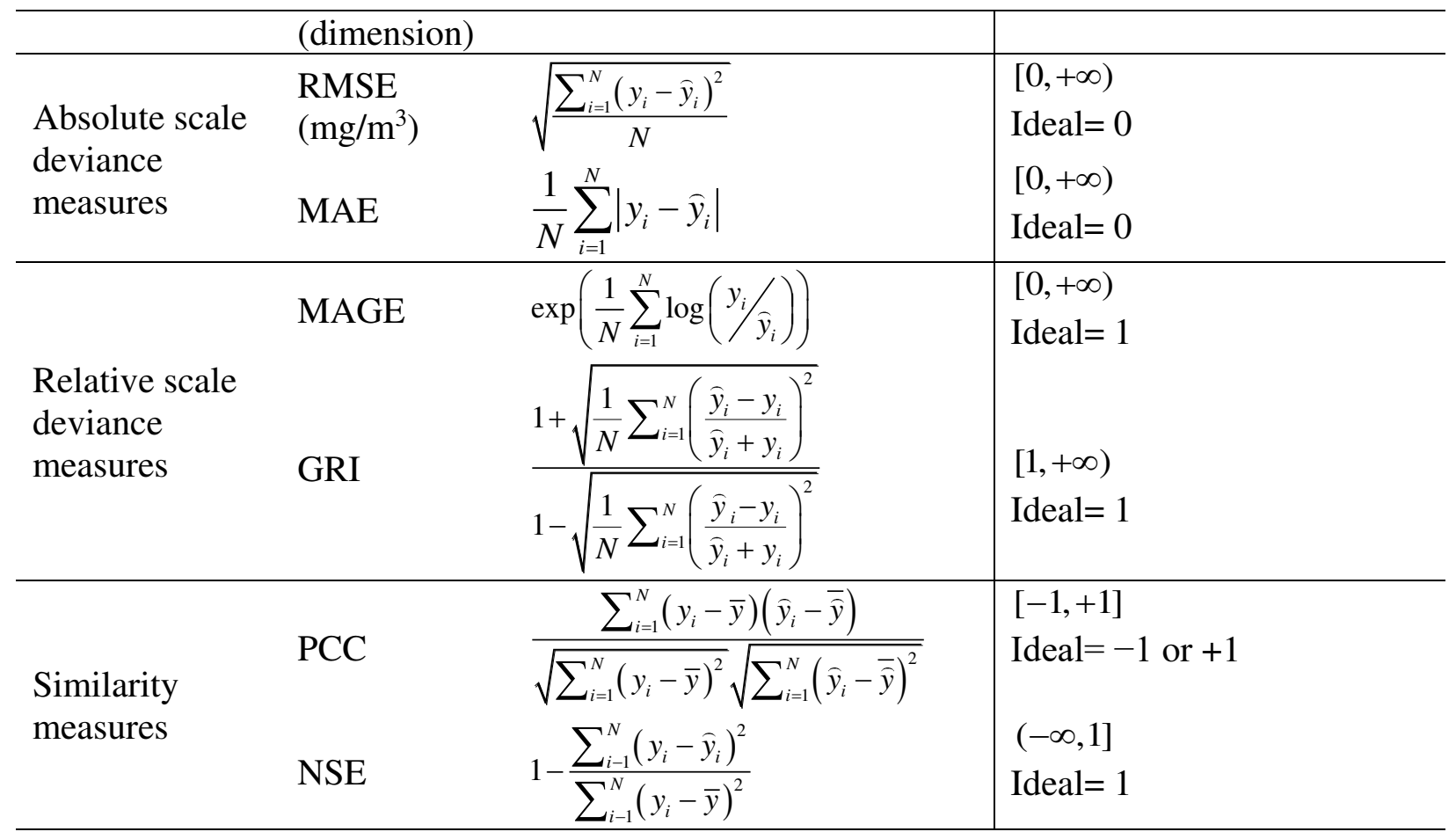

In Table 2, $y$ and $\hat{y}$ denote respectively the observed and simulated/predicted values for the influent/effluent arsenic concentrations in the training/testing stages. $\bar{y}$ is the average of the arsenic values in the desired period, and $N$ is the number of data, which stands for the number of observed values in the training or testing stages.

342

343

344

345

346

347

348

349

350

351

352

353

354

355

356

\section{Results}

In this section, the simulated and predicted outcomes of the applied models in the training and testing stages are presented for the two input combination scenarios separately.

\section{First scenario, all available input parameters}

As mentioned in Section 4.2, the first scenario considers all of the available independent parameters for the construction of the kriging and machine learning models. Table 3 shows the overall performance of the compared machine learning models in the training stage. The GBRT model shows a better training performance compared to the MARS and ANN models.

Given the results in Table 4 for the testing phase, it can be clearly seen that the kriging-logistic method excelled the absolute deviance and similarity measures at predicting effluent arsenic values in comparison to the other applied models. However, all models acted similarly for the relative deviance measures (MAGE and GRI). To be specific, the kriging-logistic method performed better than the other models for all of the statistic measures with the exception of the MAGE measure (1.009 vs. 1.006). By and large, the kriging-logistic method surpassed the other models in the prediction process of effluent arsenic. 
358 Table 3. Machine learning models' performance for the first input scenario in the training phase

\begin{tabular}{lllllll}
\hline Models & \multicolumn{2}{l}{$\begin{array}{l}\text { Absolute deviance } \\
\text { measures }(\text { ug/L) }\end{array}$} & \multicolumn{2}{l}{$\begin{array}{l}\text { Relative deviance } \\
\text { measures }\end{array}$} & \multicolumn{2}{l}{ Similarity measures } \\
\cline { 2 - 7 } & RMSE & MAE & MAGE & GRI & PCC & NSE \\
\hline MARS & 0.099 & 0.237 & 0.997 & 1.131 & 0.957 & 0.719 \\
\hline GBRT & 0.087 & 0.198 & 1.002 & 1.103 & 0.972 & 0.783 \\
\hline ANN & 0.098 & 0.231 & 0.997 & 1.129 & 0.958 & 0.726 \\
\hline
\end{tabular}

Table 4. Models' evaluation based on the statistical measures for the first input scenario in the 361 testing phase

\begin{tabular}{llllllll}
\hline Category & Models & \multicolumn{2}{l}{$\begin{array}{l}\text { Absolute deviance } \\
\text { measures }(\mathrm{ug} / \mathrm{L})\end{array}$} & \multicolumn{2}{l}{$\begin{array}{l}\text { Relative deviance } \\
\text { measures }\end{array}$} & \multicolumn{2}{l}{ Similarity measures } \\
\cline { 3 - 8 } & & RMSE & MAE & MAGE & GRI & PCC & NSE \\
\hline \multirow{2}{*}{$\begin{array}{l}\text { Machine } \\
\text { learning }\end{array}$} & MARS & 0.176 & 0.120 & 1.010 & 1.167 & 0.885 & 0.751 \\
\cline { 2 - 8 } & GBRT $*^{*}$ & 0.170 & 0.120 & 1.016 & 1.159 & 0.900 & 0.770 \\
\cline { 2 - 8 } & ANN & 0.176 & 0.121 & $\mathbf{1 . 0 0 4}$ & 1.171 & 0.867 & 0.749 \\
\hline \multirow{2}{*}{$\begin{array}{l}\text { Kriging } \\
\text { based }\end{array}$} & Kriging & 0.206 & 0.142 & 1.010 & 1.200 & 0.819 & 0.655 \\
\cline { 2 - 8 } & Kriging-logistic* & $\mathbf{0 . 1 3 6}$ & $\mathbf{0 . 1 0 4}$ & 1.009 & $\mathbf{1 . 1 2 1}$ & $\mathbf{0 . 9 3 0}$ & $\mathbf{0 . 8 5 0}$ \\
\hline
\end{tabular}

Note: Best obtained values are identified bold-faced; * The best predictive model; ** The best machine learning model

The scatter plots of the MARS, GBRT, kriging, and kriging-logistic models for the predicted values are shown in Figure 4. They compare the modeled effluent arsenic concentration with the measured samples by using the coefficient of determination $\left(\mathrm{R}^{2}\right)$. In these plots, by the aid of the lines of agreement (1:1 line) and $20 \%$ error lines, it is shown how close the measured data to the predicted values are. The $\mathrm{R}^{2}$ for the kriging-logistic model is 0.86 , which means the predicted results match the measured arsenic values to a fair extent.

In Figure 5, the predicted outcomes of the models, as well as the measured arsenic concentrations, are illustrated using violin plots. Similar to boxplots, violin plots are used to represent the comparison of a variable distribution (here the effluent arsenic) across various data categories. In these plots, the median, mean, interquartile range (midspread), and kernel density estimation (the body of the violin plot) are presented. Taking into account the above characteristics of the plots reveals that the kriging-logistic and ANN models performed better than the other models. Obviously, the kriging method failed to produce fair results, as shown by

378 the deformed kernel density shape and the interquartile range height in comparison to the 379 corresponding plot of the measured data. 

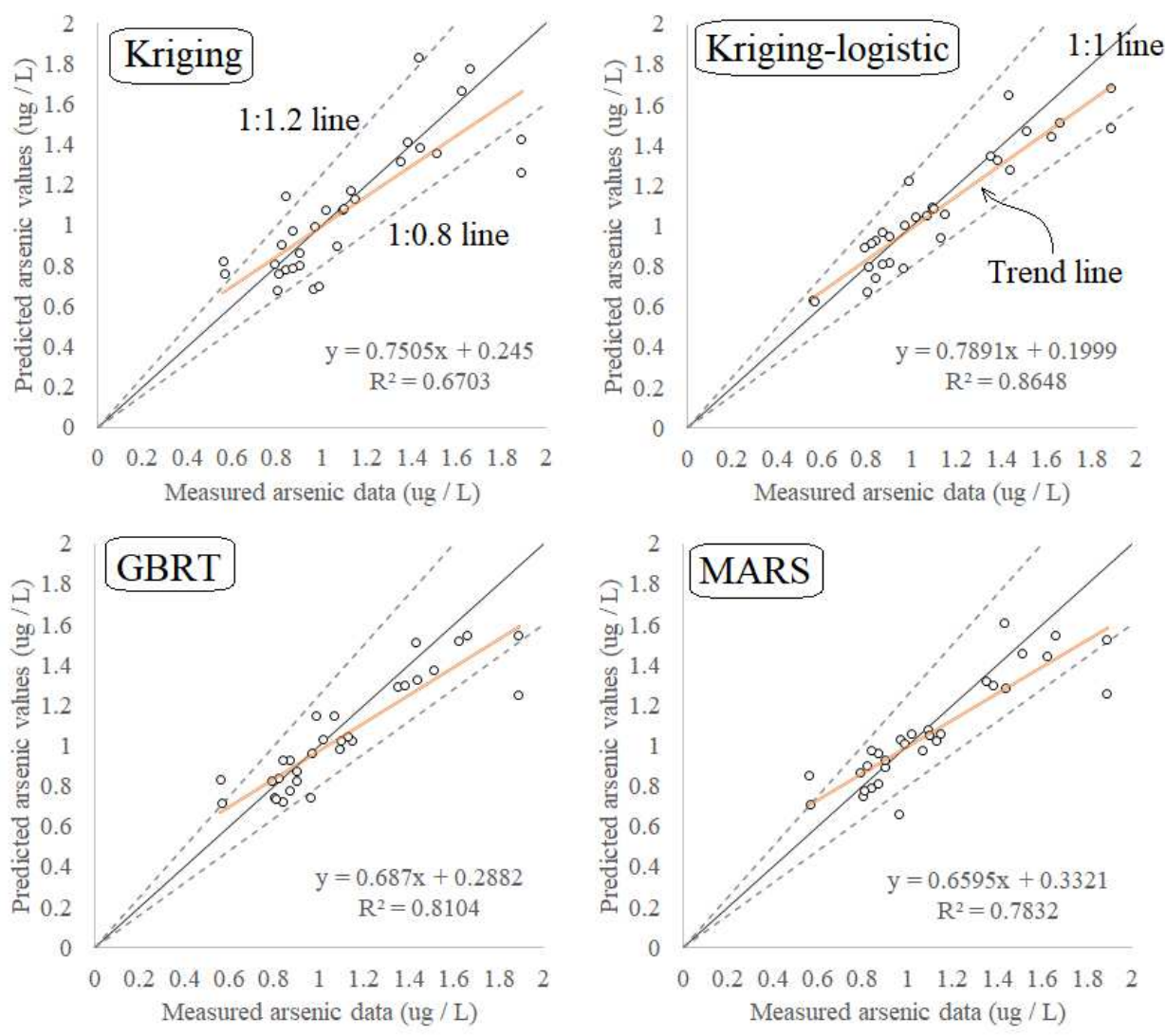

Figure 4. Scatter plots of the predicted results versus measured effluent arsenic concentration for

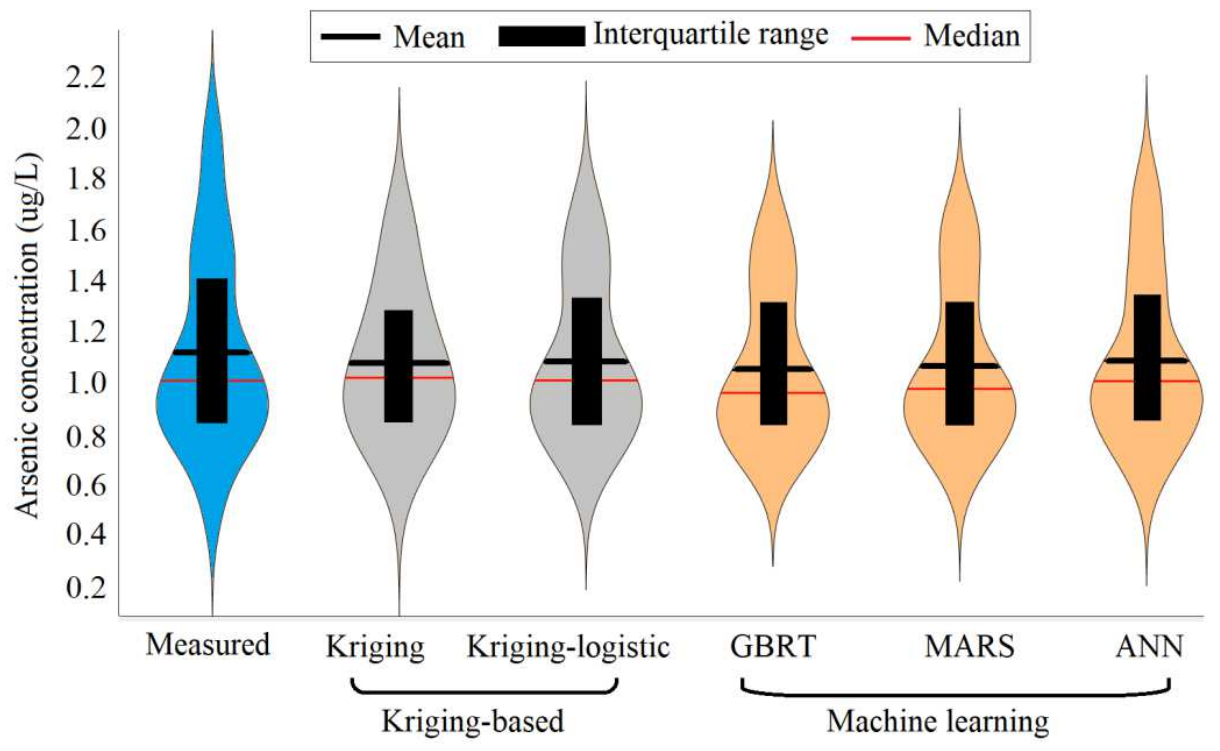

Figure 5. Violin plots of the measured versus predicted effluent arsenic by kriging-based and machine learning methods in the first modeling scenario 


\begin{tabular}{llllllll}
\hline Category & \multirow{2}{*}{\begin{tabular}{l} 
Models \\
\cline { 2 - 7 }
\end{tabular}} & & \multicolumn{2}{l}{$\begin{array}{l}\text { Absolute deviance } \\
\text { measures }(\mathrm{ug} / \mathrm{L})\end{array}$} & \multicolumn{2}{l}{$\begin{array}{l}\text { Relative deviance } \\
\text { measures }\end{array}$} & \multicolumn{2}{l}{ Similarity measures } \\
\cline { 2 - 8 } & & RMSE & MAE & MAGE & GRI & PCC & NSE \\
\hline \multirow{2}{*}{$\begin{array}{l}\text { Machine } \\
\text { learning }\end{array}$} & MARS & 0.174 & 0.113 & 1.015 & 1.167 & 0.883 & 0.757 \\
\cline { 2 - 8 } & GBRT $^{* *}$ & 0.164 & 0.113 & 1.019 & 1.157 & 0.914 & 0.787 \\
\cline { 2 - 8 } & ANN & 0.170 & 0.113 & $\mathbf{1 . 0 0 6}$ & 1.166 & 0.878 & 0.764 \\
\hline \multirow{2}{*}{$\begin{array}{l}\text { Kriging } \\
\text { based }\end{array}$} & Kriging & 0.177 & 0.126 & 1.009 & 1.181 & 0.879 & 0.747 \\
\cline { 2 - 8 } & Kriging-logistic* & $\mathbf{0 . 1 1 3}$ & $\mathbf{0 . 0 8 3}$ & 1.007 & $\mathbf{1 . 0 9 9}$ & $\mathbf{0 . 9 6 2}$ & $\mathbf{0 . 8 9 6}$ \\
\hline
\end{tabular}

Based on the calculated measures for the predicted arsenic effluent values in Table 6, it is obvious that the ANN model's performance is not as accurate as of the GBRT's. However, the highest NSE $(=0.896)$ and PCC $(=0.962)$ scores in Table 6 show that the kriging-logistic method tended to be the best-fitted model among the five at predicting effluent arsenic. Likewise, the kriging-logistic method provided the lowest values for the absolute deviance measures $(\mathrm{RMSE}=0.113 \mathrm{ug} / \mathrm{L}$ and $\mathrm{MAE}=0.083 \mathrm{ug} / \mathrm{L})$ that introduced the kriging-logistic as the most accurate model, followed by the GBRT $(\mathrm{RMSE}=0.164 \mathrm{ug} / \mathrm{L}$ and MAE $=0.113 \mathrm{ug} / \mathrm{L})$. Even though the ANN gave the best score for the MAGE, the kriging-logistic model ranked second $($ MAGE $=1.007$ ) for the MAGE and first for the GRI (= 1.099). Subsequently, the kriging-logistic model is the dominant best predictive model concerning the prediction of effluent arsenic in this study.

Table 6. Models' evaluation based on the statistical measures for the second input scenario in the testing phase

Note: Best obtained values are identified bold-faced; * The best predictive model; $* *$ The best machine learning model 
411 In order to assess the performance of the developed models graphically, scatter plots and violin 412 plots of the predicted values versus the measured data are depicted, respectively in Figures 6 and 413 7. First, the predicted effluent arsenic concentrations by the MARS, GBRT, kriging, and kriging414 logistic models are compared with the measured data in terms of scatter plots in Figure 6. In 415 addition to having a higher coefficient of determination $\left(\mathrm{R}^{2}=0.92\right)$, it can obviously be seen from 416 the provided scatter plots that the kriging-logistic method predicted values are closer to the 417 corresponding measured effluent arsenic data than those of the other applied models. Given the 418 trend line equations in the scatter plots (assume that the linear equation is $\mathrm{y}=a \mathrm{x}+b$ ), the 419 kriging-logistic method followed by the MARS model, provided closer $a$ and $b$ coefficients to 1 420 and 0 , respectively.

421
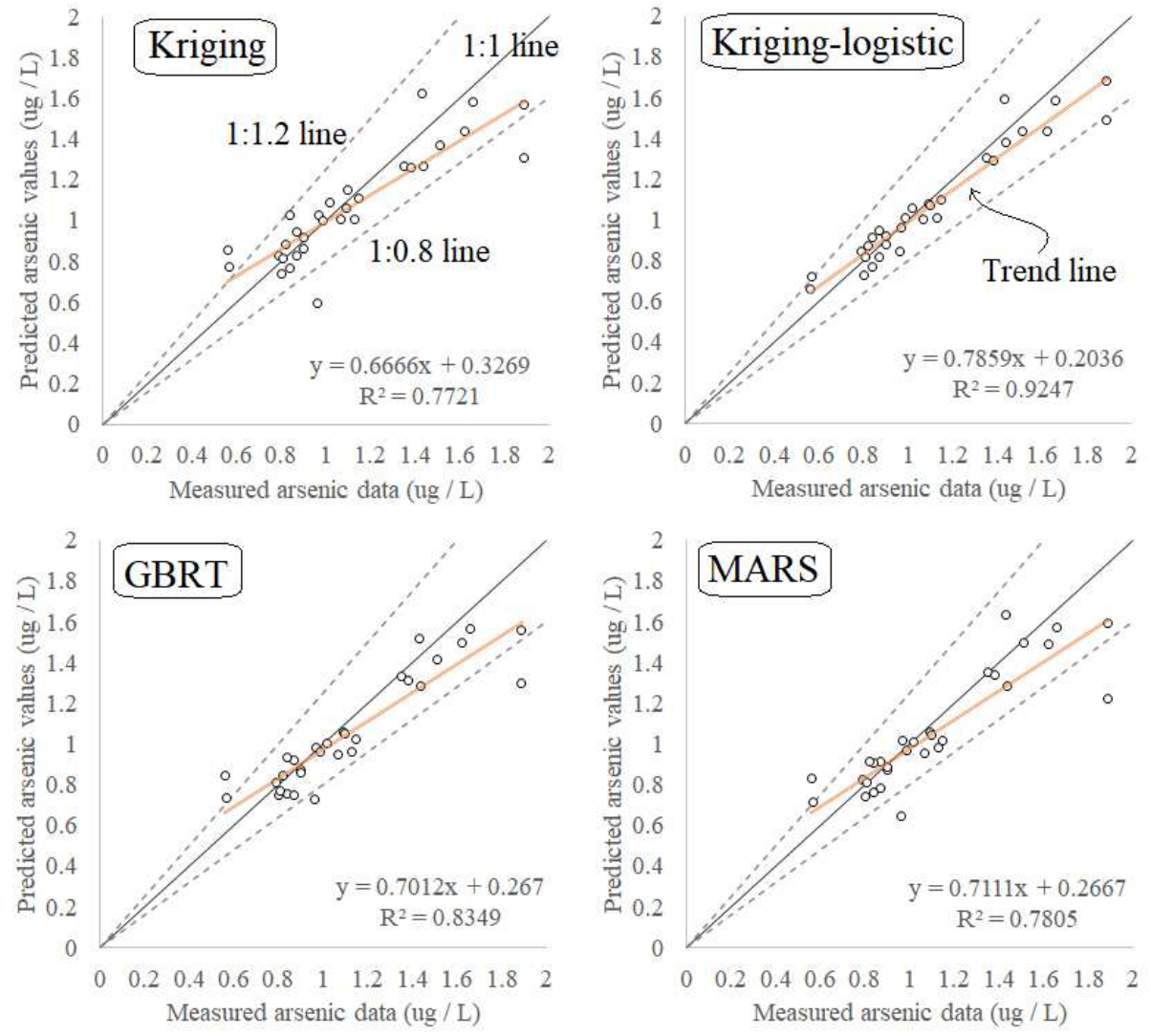

423 Figure 6. Scatter plots of the predicted results versus measured effluent arsenic concentration for 424 the second input scenario using the feature selection procedure

425 In Figure 7, the predicted results versus the measured arsenic concentration are summarized 426 graphically using violin plots. Similar to the results obtained for the first scenario, all of the 427 applied predictive models acted similarly in presenting the interquartile range. Nevertheless, the 428 ML models show better performance for the measures of central tendency, including the mean 429 and median. According to the rotated kernel density plot, it can also be easily observed that the 
predictions obtained from the kriging-logistic and ANN models are more or less the same as the rotated density plot of the measured data.

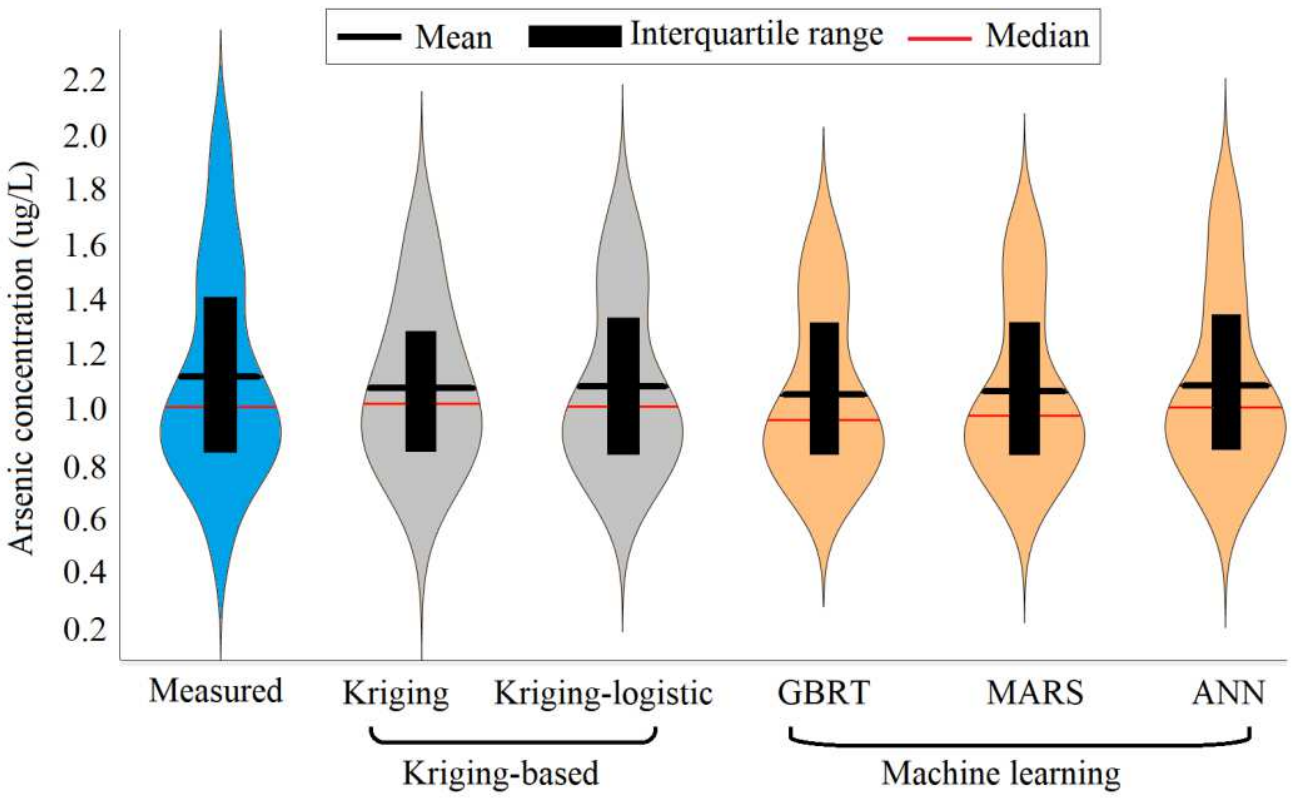

Figure 7. Violin plots of the measured versus predicted effluent arsenic by kriging-based and machine learning methods in the second modeling scenario

\section{Discussion}

In general, the ranking of the applied machine learning models is similar for both the first and second input scenarios, being overall the GBRT, then the MARS, and ANN. However, on the whole, the kriging-logistic method provided the best predictive results for both input scenarios. The simple kriging method, on the other hand, gave the weakest performance among all of the models. To seek more insight into the results of the two input scenarios, we have compared the performance of both scenarios and presented the performance enhancement of the predicted values in the second scenario compared to the first scenario in Table 7.

Table 7. The performance enhancement (\%) of the predicted values in the second scenario compared to the first scenario

\begin{tabular}{llllllll}
\hline Category & Models & \multicolumn{2}{l}{$\begin{array}{l}\text { Absolute deviance } \\
\text { measures }(\text { ug/L) }\end{array}$} & \multicolumn{2}{l}{$\begin{array}{l}\text { Relative deviance } \\
\text { measures }\end{array}$} & \multicolumn{2}{l}{ Similarity measures } \\
\cline { 3 - 8 } & & RMSE & MAE & MAGE & GRI & PCC & NSE \\
\hline \multirow{2}{*}{$\begin{array}{l}\text { Machine } \\
\text { learning }\end{array}$} & MARS & 1.14 & 5.83 & -0.50 & 0.00 & -0.23 & 0.80 \\
\cline { 2 - 8 } & GBRT** & 3.53 & 5.83 & -0.30 & 0.20 & 1.56 & 2.21 \\
\cline { 2 - 8 } & ANN & 3.41 & 6.61 & -0.20 & 0.50 & 1.27 & 2.00 \\
\hline Kriging & Kriging & 14.08 & 11.27 & 0.10 & 1.90 & 7.33 & 14.05 \\
\cline { 2 - 8 } based & Kriging-logistic* & 16.91 & 20.19 & 0.20 & 2.20 & 3.44 & 5.41 \\
\hline
\end{tabular}


As can be seen from the results in Table 7, the statistical measures related to the predicted arsenic values have been improved noticeably for the kriging methods, e.g. an averaged RMSE increase of $15 \%$. This issue emphasizes the importance of the feature selection procedure in improving the performance of the data-driven statistical-based models, such as kriging models.

A similar claim could be made for the upgrading in the performance of the applied machine learning models (with the exception of the MAGE measure). However, the magnitude of improvement is not as high (an average RMSE increase of 2.7\%) as the results of the krigingbased methods. It can be concluded that because of their complex structure, ML models are better tools in capturing the nonlinear properties of the system problem based on even some ineffective/irrelevant independent input parameters. In other words, ML models are less sensitive to the input vector parameters than the kriging methods.

\section{Conclusions}

This study assessed and evaluated the potential of kriging-based and machine learning models in predicting the effluent arsenic concentration of a treatment plant using feature selection procedure establishing two different input scenarios. To meet this end, monthly influent wastewater discharge and six quality parameters, as well as effluent arsenic concentrations, were gathered from the Point Loma WWTP, which is a primary wastewater treatment facility in San Diego, California. The performance of both model categories was evaluated in terms of statistical measures, including absolute scale deviance measures (RMSE and MAE), relative scale deviance measures (MAGE and GRI), and similarity measures (PCC and NSE), as well as diagnostic methods such as scatter and violin plots. Results showed that ML models, such as ANN, MARS, and GBRT, could be effectively applied to the monthly interval prediction of arsenic concentration of effluent. Among the ML models, overall, the GBRT model showed a slightly higher prediction accuracy in the testing stage. The standard kriging model was unsuccessful in giving acceptable predictive results compared to the other models applied. However, it was revealed that the modified kriging model, namely the kriging-logistic model, provided the best predictive results in comparison to the ML models. It is worth mentioning that the kriging-based models were more sensitive to the outcomes of the feature selection procedure than the ML models. To sum up, this study suggested the efficiency and robustness of machine learning models and the higher accuracy of kriging-logistic method in modeling effluent arsenic of WWTPs. 


\section{Authors' contributions}

480 Mohammad Zounemat-Kermani designed the study and carried out the analyses. Meysam

481 Alizamir and Behrooz Keshtegar executed the models. Mohammad Zounemat-Kermani, Okke 482 Batelaan, and Reinhard Hinkelmann wrote the paper. All authors read and approved the final 483 manuscript.

\section{$484 \quad$ Funding}

485 This work was supported by the Alexander von Humboldt Foundation under the Georg Forster 486 Research Fellowship program received by Mohammad Zounemat-Kermani as the applicant and 487 Reinhard Hinkelmann as the host.

\section{Data availability}

489 The authors confirm that the data supporting the findings of this study are available within the 490 article and its supplementary

491 materials

\section{Compliance with ethical standards}

\section{Competing interests}

494 The authors declare that they have no conflict of interest.

495 Ethics approval

496 The submitted study has not been published elsewhere in any form or language (partially or in

497 full).

498 Consent to participate and publish

499 All authors agreed with the content and all gave consent to submit and publish the research 500 paper.

\section{Acknowledgment}

502 As the corresponding author, I wish to express my appreciation to the Alexander von Humboldt 503 Foundation for providing financial support for this research project within the framework of the 504 Return Fellowship program. 


\section{References}

Alizamir, M., Kim, S., Kisi, O., \& Zounemat-Kermani, M. (2020a). A comparative study of several machine learning based non-linear regression methods in estimating solar radiation: Case studies of the USA and Turkey regions. Energy, 197, 117239.

Alizamir, M., Kim, S., Zounemat-Kermani, M., Heddam, S., Kim, N. W., \& Singh, V. P. (2020b). Kernel Extreme Learning Machine: An Efficient Model for Estimating Daily Dew Point Temperature Using Weather Data. Water, 12(9), 2600.

Altowayti, W. A. H., Haris, S. A., Almoalemi, H., Shahir, S., Zakaria, Z., \& Ibrahim, S. (2020). The removal of arsenic species from aqueous solution by indigenous microbes: Batch bioadsorption and artificial neural network model. Environmental Technology \& Innovation, 19, 100830.

Ansari, M., Othman, F., Abunama, T., \& El-Shafie, A. (2018). Analysing the accuracy of machine learning techniques to develop an integrated influent time series model: case study of a sewage treatment plant, Malaysia. Environmental Science and Pollution Research, 25(12), 12139-12149.

Bekkari, N., \& Zeddouri, A. (2019). Using artificial neural network for predicting and controlling the effluent chemical oxygen demand in wastewater treatment plant. Management of Environmental Quality: An International Journal.

Bernardelli, A., Marsili-Libelli, S., Manzini, A., Stancari, S., Tardini, G., Montanari, D., ... \& Venier, S. (2020). Real-time model predictive control of a wastewater treatment plant based on machine learning. Water Science and Technology, 81(11), 2391-2400.

Cameron, T. G., Jackel, B., \& Oliveira, D. M. (2019). Using mutual information to determine geoeffectiveness of solar wind phase fronts with different front orientations. Journal of Geophysical Research: Space Physics, 124(3), 1582-1592.

Comas, J., Dzeroski, S., Gibert, K., R-Roda, I., \& Sànchez-Marrè, M. (2001). Knowledge discovery by means of inductive methods in wastewater treatment plant data. $A I$ communications, 14(1), 45-62.

Fard, M. B., Mirbagheri, S. A., Pendashteh, A., \& Alavi, J. (2020). Estimation of effluent parameters of slaughterhouse wastewater treatment with artificial neural network and B-spline quasi interpolation. International Journal of Environmental Research, 14(5), 527-539.

Friedman, J. H. (1991). Multivariate adaptive regression splines. The annals of statistics, 1-67.

Friedman, J. H. (2001). Greedy function approximation: a gradient boosting machine. Annals of statistics, 29(5), 1189-1232.

Friedman, J. H., \& Roosen, C. B. (1995). An introduction to multivariate adaptive regression splines. Statistical Methods in Medical Research, 4(3), 197-217 
541 Gardner, M. W., \& Dorling, S. R. (1998). Artificial neural networks (the multilayer 542 perceptron) - a review of applications in the atmospheric sciences. Atmospheric environment, 543 32(14-15), 2627-2636.

544 Ge, J., Guha, B., Lippincott, L., Cach, S., Wei, J., Su, T. L., \& Meng, X. (2020). Challenges of 545 arsenic removal from municipal wastewater by coagulation with ferric chloride and alum. 546 Science of The Total Environment, 725, 138351.

547 Giri, A. K., Patel, R. K., \& Mahapatra, S. S. (2011). Artificial neural network (ANN) approach 548 for modelling of arsenic (III) biosorption from aqueous solution by living cells of Bacillus cereus 549 biomass. Chemical Engineering Journal, 178, 15-25.

550 Granata, F., Papirio, S., Esposito, G., Gargano, R., \& De Marinis, G. (2017). Machine learning 551 algorithms for the forecasting of wastewater quality indicators. Water, 9(2), 105.

552 Guo, H., Jeong, K., Lim, J., Jo, J., Kim, Y. M., Park, J. P., ... \& Cho, K. H. (2015). Prediction of 553 effluent concentration in a wastewater treatment plant using machine learning models. Journal of 554 Environmental Sciences, 32, 90-101.

555 Haghiabi, A. H., Nasrolahi, A. H., \& Parsaie, A. (2018). Water quality prediction using machine 556 learning methods. Water Quality Research Journal, 53(1), 3-13.

557 Hastie, T., Tibshirani, R., \& Friedman, J. (2009). The elements of statistical learning: data 558 mining, inference, and prediction. Springer Science \& Business Media.

559 Heddam, S., \& Kisi, O. (2018). Modelling daily dissolved oxygen concentration using least 560 square support vector machine, multivariate adaptive regression splines and M5 model tree. 561 Journal of Hydrology, 559, 499-509.

562 Heddam, S., Keshtegar, B., \& Kisi, O. (2020). Predicting total dissolved gas concentration on a 563 daily scale using kriging interpolation, response surface method and artificial neural network: 564 Case study of Columbia river Basin Dams, USA. Natural Resources Research, 29(3), 18015651818.

566 Høibye, L., Clauson-Kaas, J., Wenzel, H., Larsen, H. F., Jacobsen, B. N., \& Dalgaard, O. (2008). 567 Sustainability assessment of advanced wastewater treatment technologies. Water Science and 568 Technology, 58(5), 963-968.

569 Huang, D., Allen, T. T., Notz, W. I., \& Miller, R. A. (2006). Sequential kriging optimization 570 using multiple-fidelity evaluations. Structural and Multidisciplinary Optimization, 32(5), 369571382.

572 Hubadillah, S. K., Othman, M. H. D., Ismail, A. F., Rahman, M. A., \& Jaafar, J. (2019). A low 573 cost hydrophobic kaolin hollow fiber membrane (h-KHFM) for arsenic removal from aqueous 574 solution via direct contact membrane distillation. Separation and Purification Technology, 214, $575 \quad 31-39$. 
Icke, O., van Es, D. M., de Koning, M. F., Wuister, J. J. G., Ng, J., Phua, K. M., ... \& Tao, G. (2020). Performance improvement of wastewater treatment processes by application of machine learning. Water Science and Technology, 82(12), 2671-2680.

Jachner, S., Van den Boogaart, G., \& Petzoldt, T. (2007). Statistical methods for the qualitative assessment of dynamic models with time delay (R Package qualV). Journal of Statistical Software, 22(8), 1-30.

Jiang, C., Qiu, H., Gao, L., Wang, D., Yang, Z., \& Chen, L. (2020). Real-time estimation errorguided active learning Kriging method for time-dependent reliability analysis. Applied Mathematical Modelling, 77, 82-98.

Keshtegar, B., Mert, C., \& Kisi, O. (2018). Comparison of four heuristic regression techniques in solar radiation modeling: Kriging method vs RSM, MARS and M5 model tree. Renewable and sustainable energy reviews, 81, 330-341.

Kim, S., Alizamir, M., Zounemat-Kermani, M., Kisi, O., \& Singh, V. P. (2020). Assessing the biochemical oxygen demand using neural networks and ensemble tree approaches in South Korea. Journal of Environmental Management, 270, 110834.

Krige, D. G. (1952). A statistical approach to some basic mine valuation problems on the Witwatersrand, by DG Krige, published in the Journal, December 1951: interim reply by the author to the discussion. Journal of the Southern African Institute of Mining and Metallurgy, 52(11), 264-266.

Lu, C., Feng, Y. W., Liem, R. P., \& Fei, C. W. (2018). Improved kriging with extremum response surface method for structural dynamic reliability and sensitivity analyses. Aerospace Science and Technology, 76, 164-175.

Mandal, S., Mahapatra, S. S., \& Patel, R. K. (2015). Neuro fuzzy approach for arsenic (III) and chromium (VI) removal from water. Journal of Water Process Engineering, 5, 58-75.

Matheron, Georges. "Principles of geostatistics." Economic geology 58.8 (1963): 1246-1266.

Meyer, H., Reudenbach, C., Hengl, T., Katurji, M., \& Nauss, T. (2018). Improving performance of spatio-temporal machine learning models using forward feature selection and target-oriented validation. Environmental Modelling \& Software, 101, 1-9.

Mohan, D., \& Pittman Jr, C. U. (2007). Arsenic removal from water/wastewater using adsorbents - a critical review. Journal of hazardous materials, 142(1-2), 1-53.

Pai, T. Y., Tsai, Y. P., Lo, H. M., Tsai, C. H., \& Lin, C. Y. (2007). Grey and neural network prediction of suspended solids and chemical oxygen demand in hospital wastewater treatment plant effluent. Computers \& Chemical Engineering, 31(10), 1272-1281.

Pai, T. Y., Wan, T. J., Hsu, S. T., Chang, T. C., Tsai, Y. P., Lin, C. Y., ... \& Yu, L. F. (2009). Using fuzzy inference system to improve neural network for predicting hospital wastewater treatment plant effluent. Computers \& Chemical Engineering, 33(7), 1272-1278. 
612 Plewniak, F., Crognale, S., Rossetti, S., \& Bertin, P. N. (2018). A genomic outlook on 613 bioremediation: the case of arsenic removal. Frontiers in microbiology, 9, 820.

614 Pretreatment Annual Report for the Point Loma POTW. (2009-2019) The city of San Diego, 615 public utilities department, Environmental Monitoring and Technical Services Division, 616 https://www.sandiego.gov

617 Qin, X., Gao, F., \& Chen, G. (2012). Wastewater quality monitoring system using sensor fusion 618 and machine learning techniques. Water research, 46(4), 1133-1144.

619 Rodriguez-Galiano, V. F., Luque-Espinar, J. A., Chica-Olmo, M., \& Mendes, M. P. (2018). 620 Feature selection approaches for predictive modelling of groundwater nitrate pollution: An 621 evaluation of filters, embedded and wrapper methods. Science of the total environment, 624, $622 \quad 661-672$.

623 Sakata, S., Ashida, F., \& Zako, M. (2003). Structural optimization using Kriging approximation. 624 Computer methods in applied mechanics and engineering, 192(7-8), 923-939.

625 Samadi, S. H., Ghobadian, B., \& Nosrati, M. (2021). Prediction of higher heating value of 626 627

628 629 630 biomass materials based on proximate analysis using gradient boosted regression trees method. Energy Sources, Part A: Recovery, Utilization, and Environmental Effects, 43(6), 672-681.

Sharafati, A., Asadollah, S. B. H. S., \& Hosseinzadeh, M. (2020). The potential of new ensemble machine learning models for effluent quality parameters prediction and related uncertainty. Process Safety and Environmental Protection, 140, 68-78.

Sheikhi, S., Faraji, Z., \& Aslani, H. (2021). Arsenic health risk assessment and the evaluation of groundwater quality using GWQI and multivariate statistical analysis in rural areas, Hashtroud, Iran. Environmental Science and Pollution Research, 28(3), 3617-3631.

637 Sun, Z., Wang, J., Li, R., \& Tong, C. (2017). LIF: A new Kriging based learning function and its 638 application to structural reliability analysis. Reliability Engineering \& System Safety, 157, 152639165.

640 Wang, A., Zhou, K., Zhang, X., Zhou, D., Peng, C., \& Chen, W. (2020). Arsenic removal from 641 highly-acidic wastewater with high arsenic content by copper-chloride synergistic reduction. 642 Chemosphere, 238, 124675.

643 Yun, W., Lu, Z., \& Jiang, X. (2018). An efficient reliability analysis method combining adaptive 644 Kriging and modified importance sampling for small failure probability. Structural and 645 Multidisciplinary Optimization, 58(4), 1383-1393.

646 Zhang, M., Li, Y., Long, X., Chong, Y., Yu, G., \& He, Z. (2018). An alternative approach for 647 nitrate and arsenic removal from wastewater via a nitrate-dependent ferrous oxidation process. 648 Journal of environmental management, 220, 246-252. 
649 Zhang, W., \& Goh, A. T. (2016). Multivariate adaptive regression splines and neural network 650 models for prediction of pile drivability. Geoscience Frontiers, 7(1), 45-52.

651 Zhou, Z., Liu, Y. G., Liu, S. B., Liu, H. Y., Zeng, G. M., Tan, X. F., ... \& Cai, X. X. (2017). 652 Sorption performance and mechanisms of arsenic (V) removal by magnetic gelatin-modified 653 biochar. Chemical Engineering Journal, 314, 223-231.

654 Zounemat-Kermani, M., Beheshti, A. A., Ataie-Ashtiani, B., \& Sabbagh-Yazdi, S. R. (2009). 655 Estimation of current-induced scour depth around pile groups using neural network and adaptive 656 neuro-fuzzy inference system. Applied Soft Computing, 9(2), 746-755.

657 Zounemat-Kermani, M., Matta, E., Cominola, A., Xia, X., Zhang, Q., Liang, Q., \& Hinkelmann, 658 R. (2020). Neurocomputing in Surface Water Hydrology and Hydraulics: A Review of Two 659 Decades Retrospective, Current Status and Future Prospects. Journal of Hydrology, 585, 125085.

660 Zounemat-Kermani, M., Stephan, D., \& Hinkelmann, R. (2019). Multivariate NARX neural 661 network in prediction gaseous emissions within the influent chamber of wastewater treatment 662 plants. Atmospheric Pollution Research, 10(6), 1812-1822. 


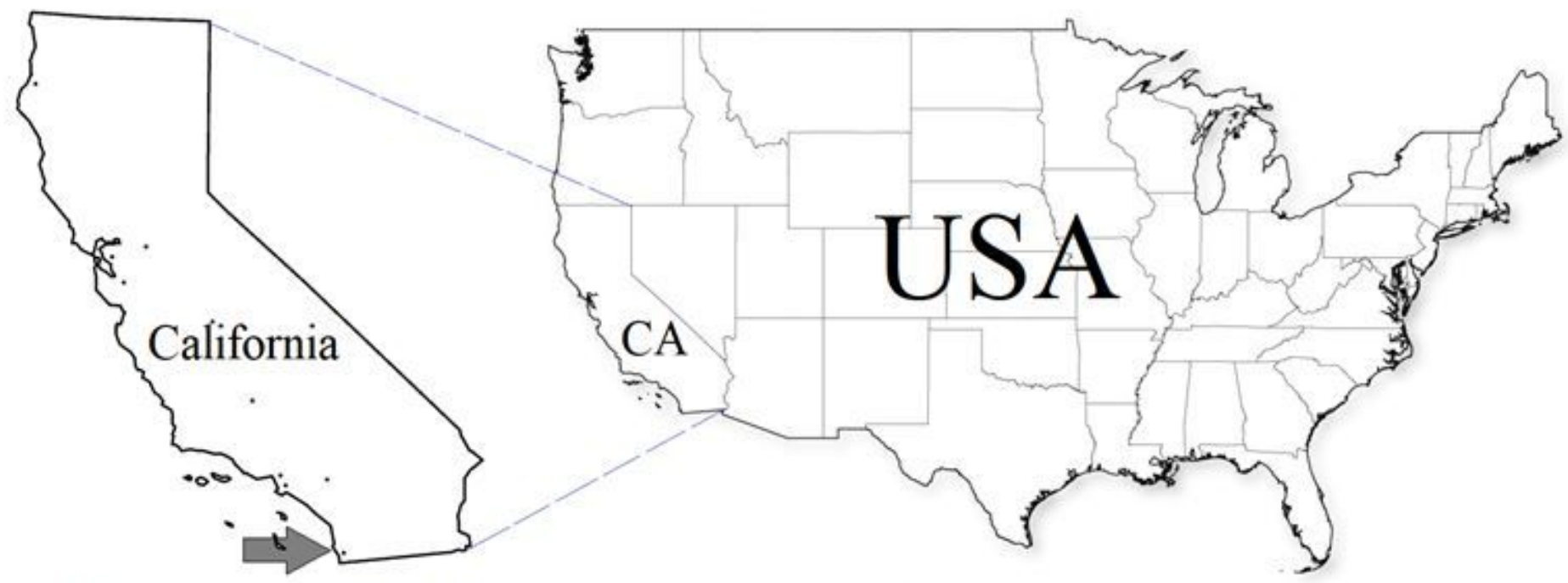

Point Loma WWTP

Figure 1

Location of the Point Loma WWTP Note: The designations employed and the presentation of the material on this map do not imply the expression of any opinion whatsoever on the part of Research Square concerning the legal status of any country, territory, city or area or of its authorities, or concerning the delimitation of its frontiers or boundaries. This map has been provided by the authors.

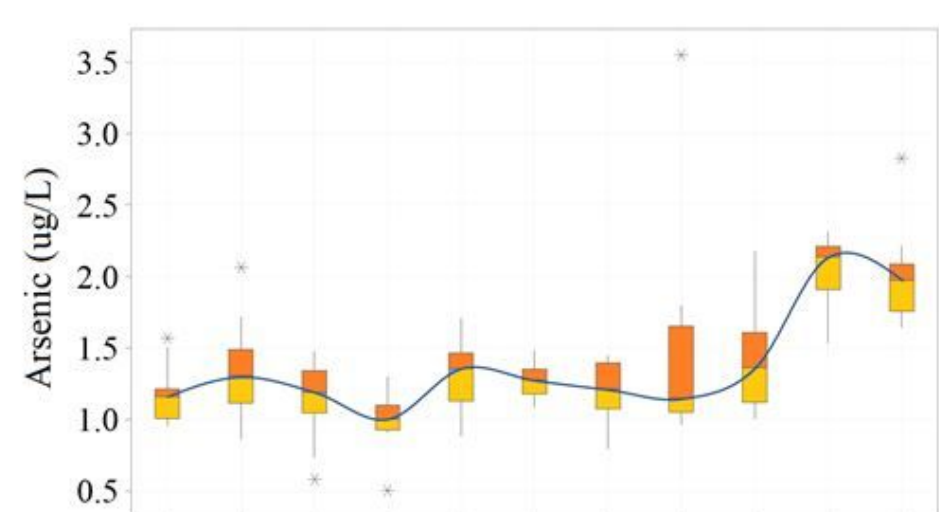

(a) $\nu^{0^{9}} \nu^{0^{0}} \nu^{0^{\prime}} \nu^{0^{2}} \nu^{0^{3}} \nu^{0^{x}} \nu^{15} \nu^{0^{6}} \nu^{1} \nu^{10} \nu^{0^{9}}$

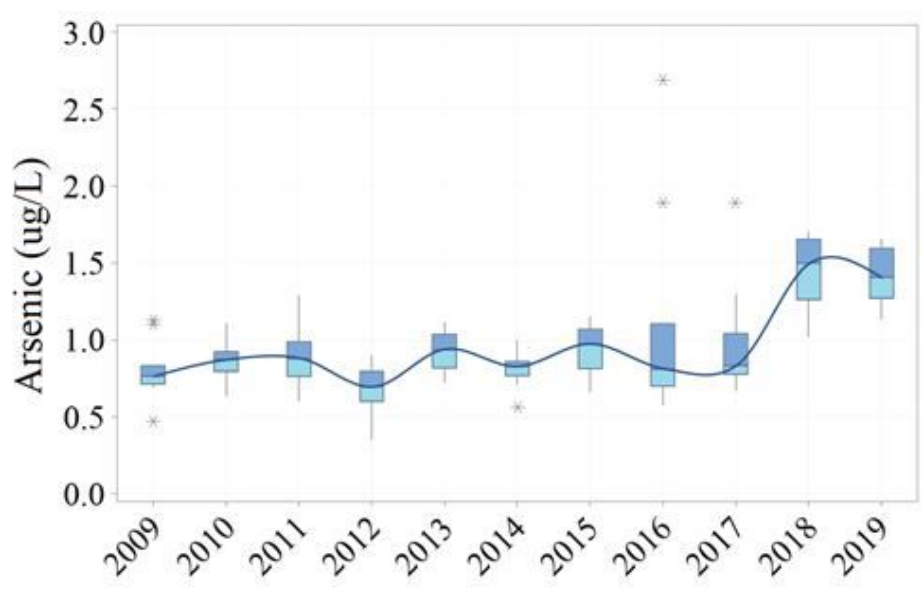

Figure 2

Annual variations of the arsenic concentrations using boxplots based on the monthly datasets; (a) influent and (b) effluent; (asterisks represent the outlier values) 


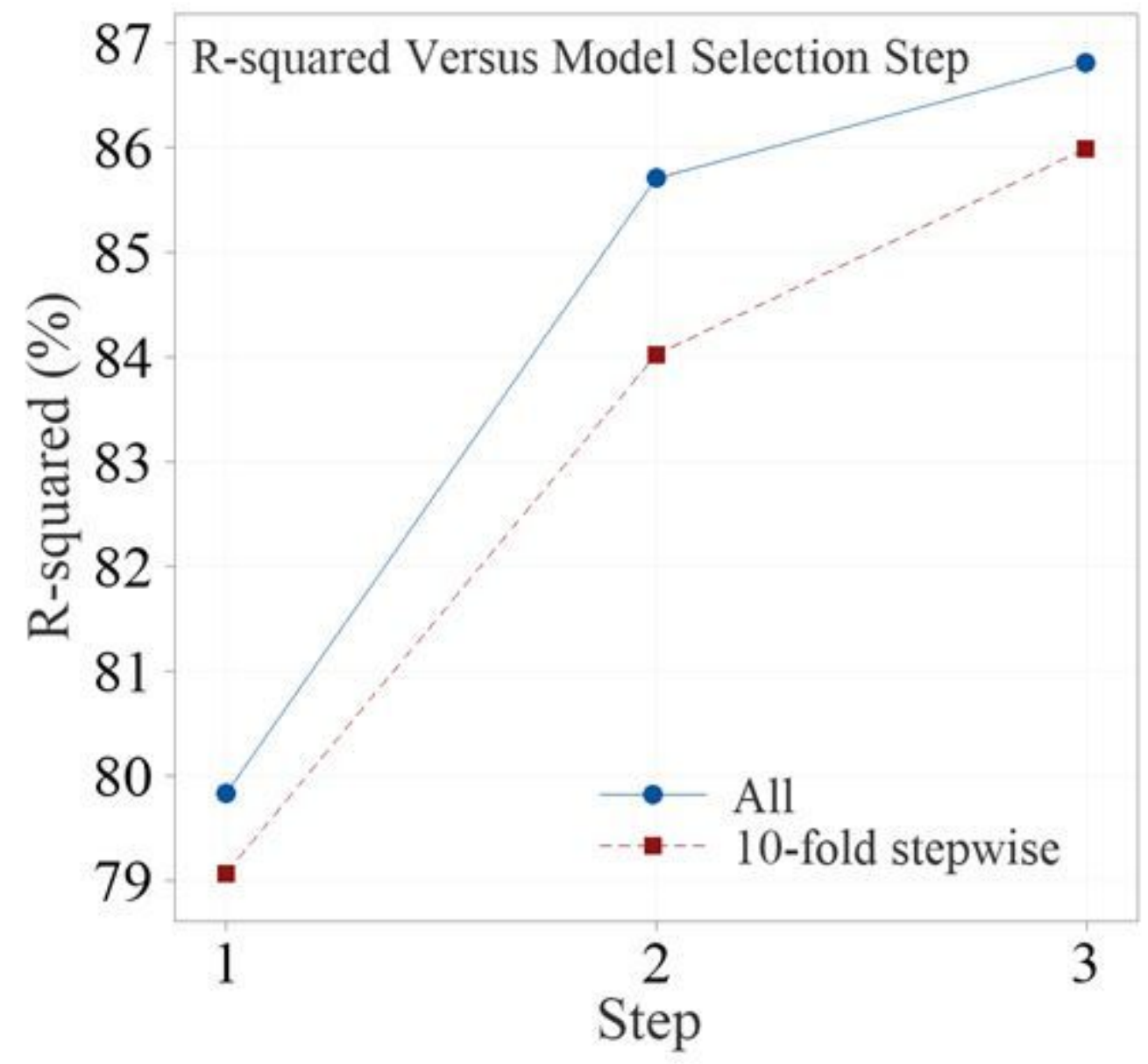

Figure 3

The result of forward feature selection analysis with 10-fold cross-validation leading to select 3 effective input parameters (flow rate, influent arsenic, and temperature) out of the 7 available variables (see Table 1) 

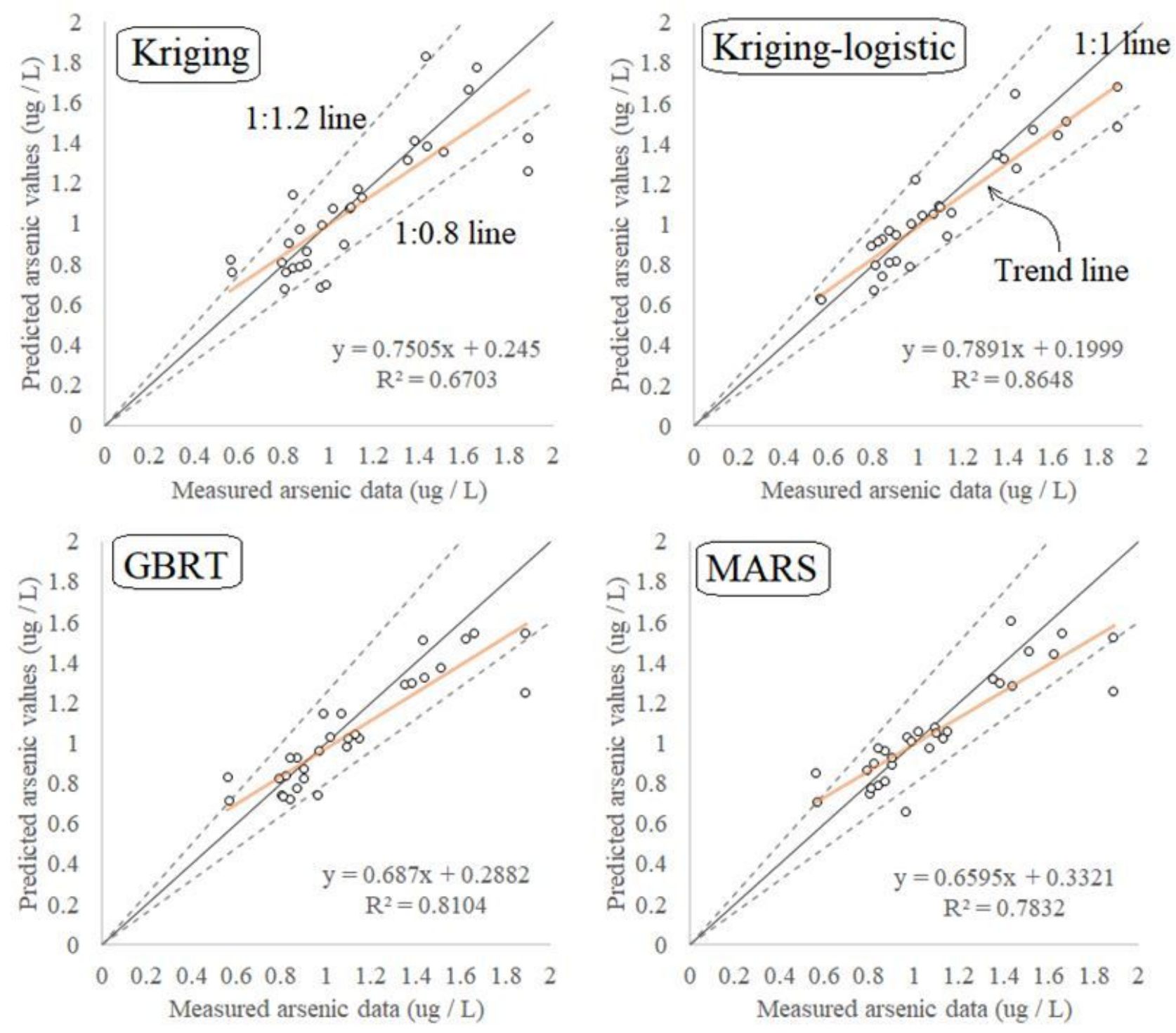

Figure 4

Scatter plots of the predicted results versus measured effluent arsenic concentration for the first input scenario 


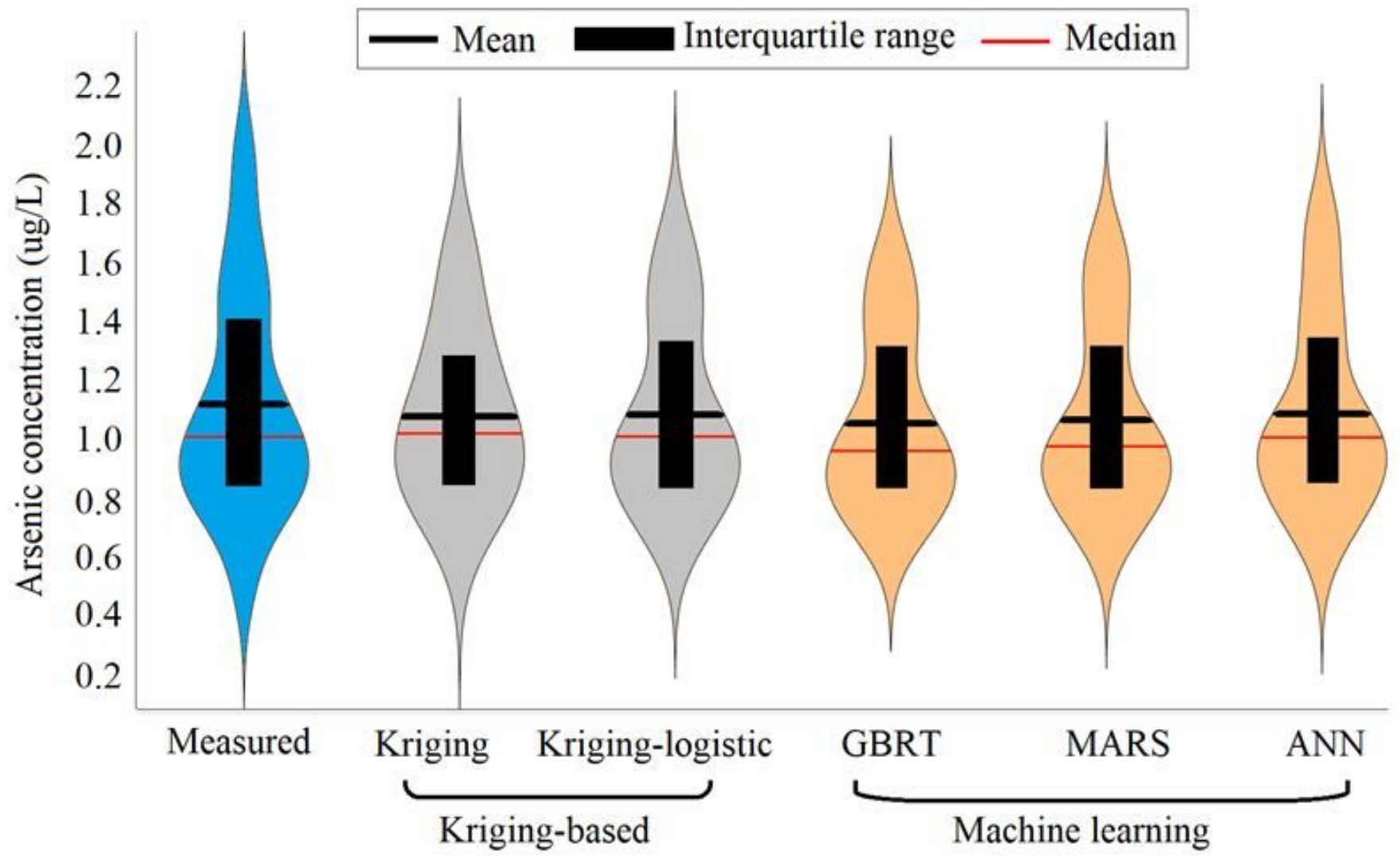

Figure 5

Violin plots of the measured versus predicted effluent arsenic by kriging-based and machine learning methods in the first modeling scenario 

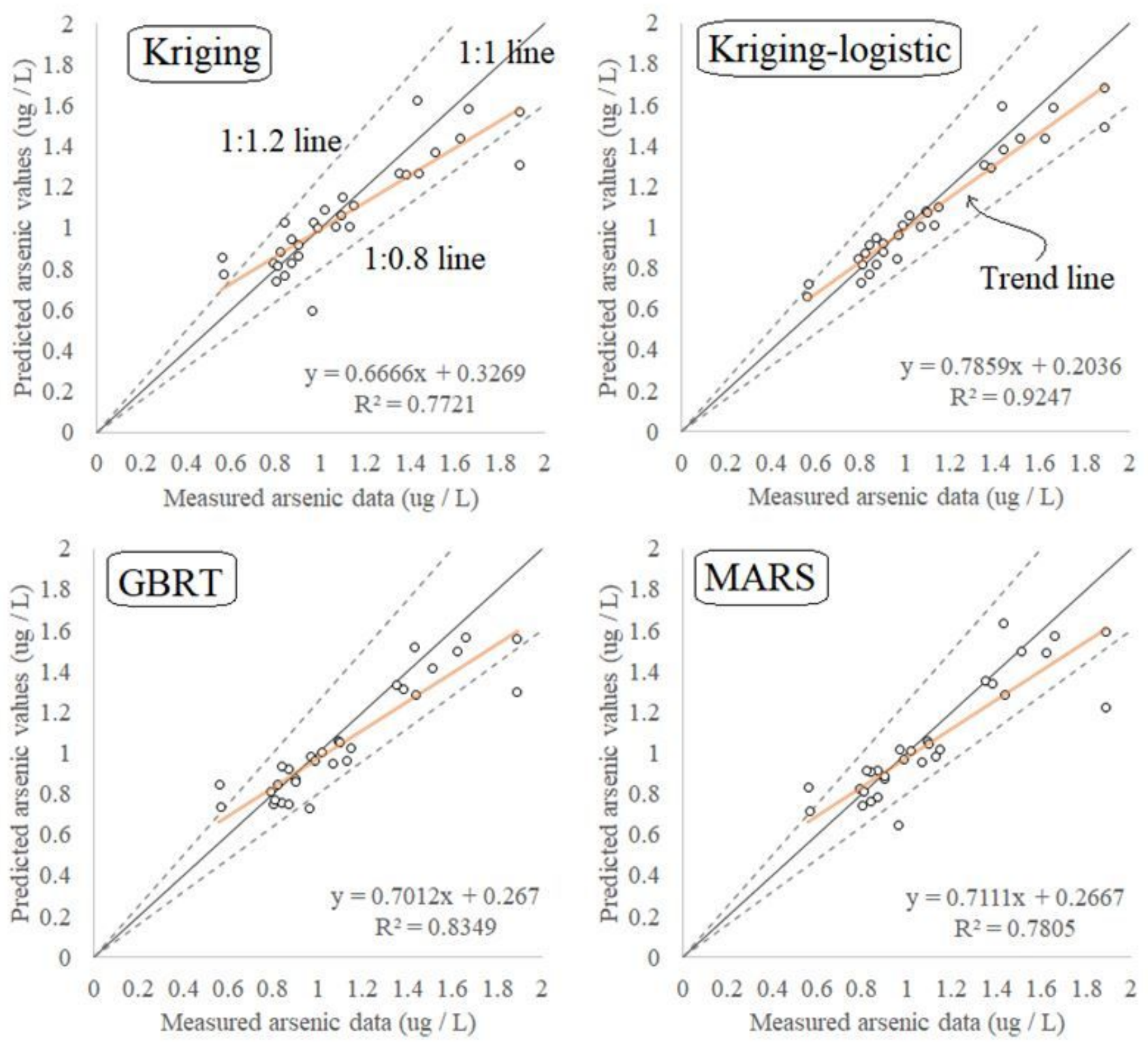

Figure 6

Scatter plots of the predicted results versus measured effluent arsenic concentration for the second input scenario using the feature selection procedure 


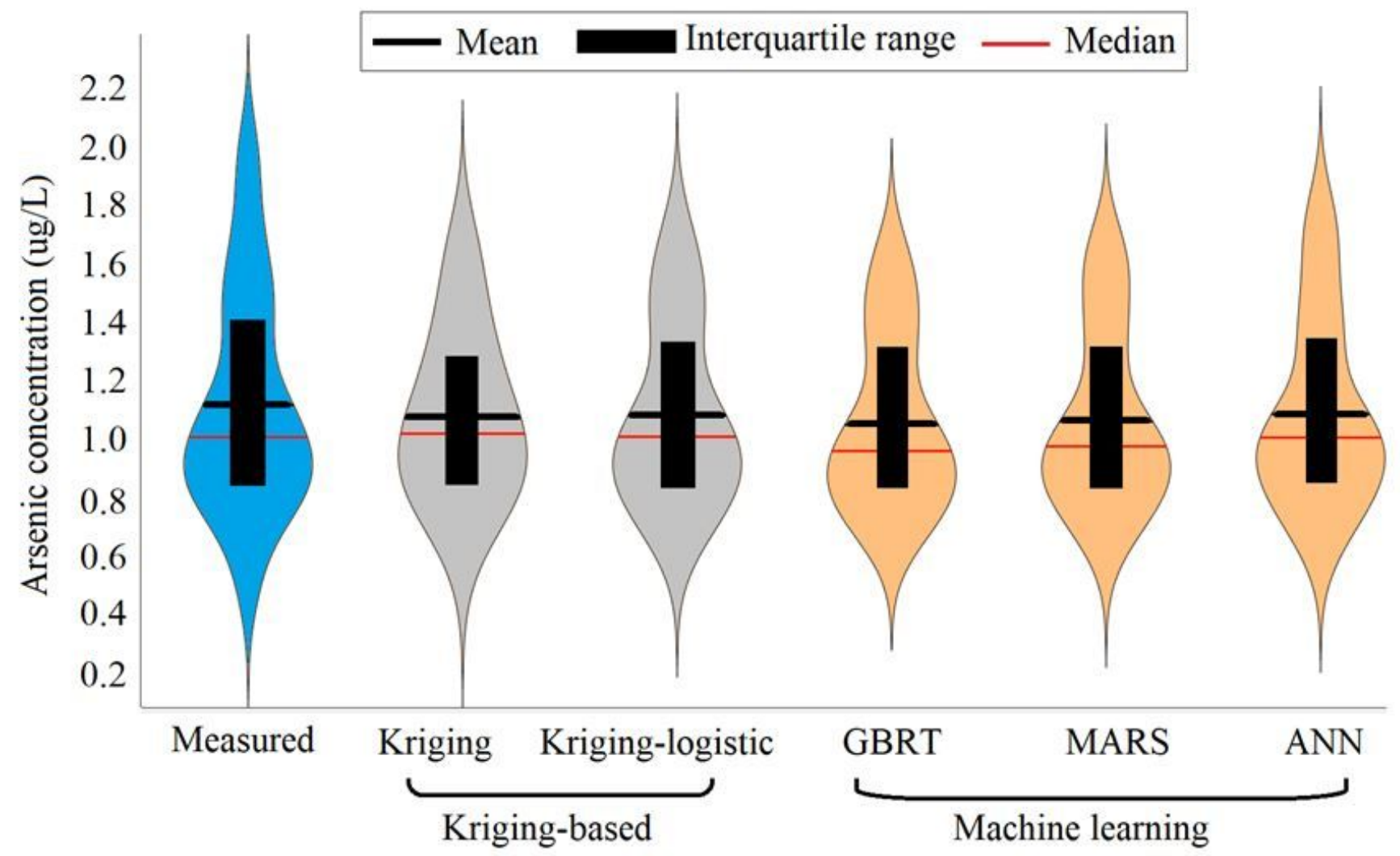

Figure 7

Violin plots of the measured versus predicted effluent arsenic by kriging-based and machine learning methods in the second modeling scenario 